\title{
Impacto fisiológico de la musicoterapia en la depresión, ansiedad, y bienestar del paciente con demencia tipo Alzheimer. Valoración de la utilización de cuestionarios para cuantificarlo
}

\author{
José Enrique de la Rubia Ortí, Paula Sancho Espinós y Carmen \\ Cabañés Iranzo \\ Universidad Católica de Valencia (España)
}

\begin{abstract}
La enfermedad de Alzheimer se ha convertido en una de las dolencias más prevalentes y que más preocupa en nuestra sociedad por el impacto que genera tanto en el paciente como en familiares y cuidadores. Debido en gran medida a que los tratamientos farmacológicos no son a día de hoy curativos de esta demencia, nos planteamos terapias alternativas que mejoren todos los parámetros de la enfermedad. En este sentido, se conoce la relación directa entre el estrés crónico que sufre el individuo en los últimos años de su vida, y la aparición de los primeros síntomas de la enfermedad destacando la depresión, ansiedad y tristeza. Y al pensar en los extraordinarios beneficios que tiene la música, nos planteamos valorar el impacto que tendría la musicoterapia en estos síntomas. Para ello, realizamos una intervención corta aplicando un protocolo estandarizado por nosotros. Paralelamente, realizamos cuestionarios estandarizados que miden la depresión y la ansiedad relacionadas con el estrés, y la felicidad relacionada con el bienestar, antes y después de la intervención, pudiendo valorar si hay mejoría, y si el empleo de los cuestionarios es adecuado para cuantificarla. Los resultados obtenidos muestran una gran mejora en los pacientes en relación a estos síntomas, pudiendo considerar por tanto a la musicoterapia como una herramienta terapéutica muy eficaz para disminuir el estrés de estos pacientes.
\end{abstract}

Palabras clave: Alzheimer, musicoterapia, estrés, depresión, ansiedad, bienestar.

Physiological impact of music therapy on depression, anxiety and well-being of patients with dementia of Alzheimer's type. Assessment of the use of questionnaires to quantify it. Alzheimer's disease has become one of the most prevalent and worrying diseases in our society due to the impact that it has on the patient as well as family members and caregivers. Largely due to the fact that nowadays pharmacological treatments are not curative of this dementia, we considered alternative therapies that improve all the parameters of the disease. In this respect, it is known the direct relationship between chronic stress experienced by the individual in the last years of their life and the appearance of the first symptoms of the disease, standing out depression, anxiety and sadness. Thinking about the extraordinary benefits that music has, we set out to assess the impact that music therapy would have on these symptoms. For this purpose, we carried out a brief intervention using a protocol standardized by us. At the same time, we conducted standardized questionnaires that measure depression and anxiety related to stress and happiness related to well-being, before and after the intervention, being able to assess whether there is any improvement, and if the use of questionnaires is suitable to quantify it. The results show a great improvement in patients regarding these symptoms, and therefore music therapy can be considered to be a very effective therapeutic tool to reduce the stress in these patients.

Keywords: Alzheimer, music therapy, stress, depression, anxiety, well-being.

Correspondencia: José Enrique de la Rubia Ortí. C/ Emilio Lluch 19, 6. C.P: 46014. Valencia (España). E-mail: joseenrique.delarubi@ucv.es 


\section{Demencia y Alzheimer}

Con el envejecimiento aparecen diversas patologías pero de entre todas, seguramente sea la demencia una de las que más problemas generan, ya no sólo al que la padece sino también a su cuidador principal y familiares. Esto es debido al deterioro tanto cognitivo como motor que sufren estos pacientes y que condiciona en gran medida su calidad de vida (Rodríguez, Ortiz y Schwochert, 2012).

De las demencias, a día de hoy el Alzheimer es la más padecida en la población. Se trata de una enfermedad degenerativa crónica caracterizada por una pérdida de la identidad personal y social (Sagasti y Bernaola, 1998). Podemos definir la Enfermedad del Alzheimer como "demencia degenerativa primaria de predominio cortical e inicio insidioso a partir de los cincuenta años, progresiva e irreversible, caracterizada por pérdida de diversas capacidades intelectuales y cognitivas, lo que conduce a un comportamiento alterado, con pérdida de los hábitos de cuidado personal, deterioro de la relación con las personas y con el entorno, y trastornos neurológico y fisicos diversos" (Sarasa, 2009).

La enfermedad presenta tres estadios, cada uno con diversos síntomas y signos. A grandes rasgos hay un deterioro en la memoria como signo principal a corto y medio plazo, seguido de una pérdida de autonomía, y finalizando con una pérdida de las actividades diarias (Tárraga, 1998).

Sin embargo, la evolución de la enfermedad no siempre es la misma. Puede ser de una forma rápida o lenta, incluso puede que haya una mezcla de signos y síntomas de los diferentes estadios. En todo caso, finalmente hay una alteración funcional a nivel cognitivo (lenguaje, percepción), motor (habilidades básicas), y en la conducta (depresión, ansiedad, trastorno del sueño, irritabilidad) (Sagasti y Bernaola, 1998).

A esto ello debemos sumarle los problemas de autoestima que afectan al estado del enfermo y sus familiares, entre los cuales destacan: dependencia, sentimientos de inutilidad, cansancio del cuidador principal, etc. (Montés, 2013).

\section{Terapias frente al Alzheimer}

En la actualidad no existe tratamiento curativo con respecto a ésta enfermedad y la mejoría clínica conseguida con tratamientos farmacológicos es mínima. Por ello, la utilización de terapias complementarias, puede ser beneficiosa para una mejora del bienestar del paciente (Montés, 2013).

Mediante las terapias se pretende reducir el impacto de la enfermedad, enlentecer el curso de la misma, retardar el deterioro cognitivo y controlar tanto la conducta como la aparición de complicaciones (Sánchez, Castro, Herrera y Acuñas, 2013).

A la hora de clasificar las terapias se utiliza la categorización APA (1997): terapias con enfoque emocional; terapias de aproximación conductual; terapias de 
enfoque cognitivo; terapias de estimulación y terapias dirigidas a los cuidadores (Montés, 2013).

\section{Musicoterapia y sus aplicaciones}

Recientemente se está empezando a instaurar en diferentes hospitales, residencias, centros y asociaciones, la musicoterapia como una posible alternativa para tratar un gran abanico de patologías (Castro, 2005). Para comprender de una forma correcta lo que supone la musicoterapia, tendremos que detenernos previamente en los conceptos de música y terapia. En primer lugar es importante saber qué significa la música, y qué es lo que representa. La música es un hecho cultural integrado en todas las actividades del ser humano. Pedagógicamente tiene un potencial muy elevado conectando lo sensorial, lo intelectivo y lo volitivo. Su valor varía según la intervención de los factores emocionales, fisiológicos, culturales o técnicos habiendo un orden matemático (Andrés, 2000).

La música es el arte de los sonidos, y por tanto implica tanto a la expresión como a la percepción. Johan Huizinga la define como un "acto libre, autosuficiente, definido, serio, sugestivo, extraordinario, alegre, impulsivo e incierto” (Andrés, 2000).

La música se ha difundido por el ser humano, llegando a estar en todas las culturas. Podemos decir que "no hay otra actividad cultural humana que sea tan penetrante como la música, la cual llega, moldea, y a menudo controla muchas de las conductas humanas" (Davis, Gfeller y Thaut, 2000).

En cuanto a la terapia tenemos que decir que hay diferentes aplicaciones de ella. En éste caso una de las intervenciones que nos interesa son las terapias creativas y artísticas. La terapia artística es una combinación entre arte y psicoterapia en donde cada una de estas partes se ve estimulada en su unión con la otra (Díez, 1996). En éste tipo de terapias podemos encontrar la arteterapia, la musicoterapia, la dramaterapia, la danzaterapia y el psicodrama (Augé, 2000).

La musicoterapia tiene muchas definiciones. Según la NAMT (National Association for Music Therapy):

"La musicoterapia es la utilización de la música para conseguir objetivos terapéuticos: la restauración, mantenimiento y mejora de la salud mental y física. Es la aplicación sistemática de la música, dirigida por un musicoterapeuta en un contexto terapéutico a fin de facilitar cambios en la conducta. Estos cambios ayudan a que el individuo en terapia se entienda mejor a sí mismo y a su propio mundo, llegando así a adaptarse mejor a la sociedad (Augé, 2000).

Para completar el concepto de musicoterapia, a continuación mostramos la definición de la World Federation of Music Therapy (WFMT):

"La musicoterapia consiste en el uso de la música y/o de sus elementos musicales (sonido, ritmo, melodía, armonía) por un musicoterapeuta, con un paciente o 
grupo, en el proceso diseñado para facilitar y promover la comunicación, el aprendizaje, la movilización, la expresión, la organización u otros objetivos terapéuticos relevantes, con el fin de lograr cambios y satisfacer necesidades física, emocionales, mentales, sociales y cognitivas. La musicoterapia busca descubrir potenciales y restituir funciones del individuo para que éste alcance una mejor organización intra e interpersonal y, consecuentemente una mejor calidad de vida a través de la prevención y rehabilitación en un tratamiento “ (Soria-Urios, Duque y Moreno, 2011).

Hay diferentes formas de la aplicación de la musicoterapia de forma terapéutica (Castro, 2005):

Musicoterapia pasiva: la persona escucha y la música persuade sin ninguna utilización de instrumentos ni movimientos.

Musicoterapia activa: es una forma más participativa. El paciente canta o emite sonidos, realiza movimientos, compone...

Musicoterapia mixta: es una mezcla de las dos terapias anteriores.

Musicoterapia receptiva: resultado de la escucha del paciente. (música vibroacústica).

Musicoterapia creativa: permite la creación de música mediante ritmos que el paciente compone mediante la voz, instrumentos, incluso los movimientos corporales.

La musicoterapia es utilizada actualmente en diversas patologías, sobre todo para una estimulación cognitiva y una mejora de la calidad de las personas y cuidadores implicados, incluso para crear un ambiente de relajación. Entre otras podemos destacar las siguientes (Soria-Urios, Duque y Moreno, 2011):

Depresión: la depresión conlleva un gran impacto tanto emocional como físico. Para tratarla se ha utilizado terapias tradicionales junto con la musicoterapia, para una mayor reducción de los síntomas.

Esquizofrenia: siendo una enfermedad psiquiátrica requiere un tratamiento farmacológico. Realizando un número de sesiones adecuado se puede lograr una mejor en el estado global y mental y su funcionamiento, además de una clara mejoría en las relaciones interpersonales.

Afasia: las personas con afasia no fluida pueden llegar a cantar palabras aunque no puedan hablar. En estos pacientes se juega con la utilización de la melodía y el ritmo, pudiendo mejorar su producción silábica.

Autismo: sabemos que uno de los grandes problemas del autismo es la comunicación y las relaciones sociales. Con la música se llega a conseguir una estimulación en la comunicación y expresión. Ésta es una de las patologías donde más se utiliza ésta terapia.

Daño cerebral: "la terapia neurológica musical se define como la aplicación terapéutica de la música en disfunciones motoras, sensoriales y cognitivas secundarias a alteraciones neurológicas". 


\section{Musicoterapia y Alzheimer}

La musicoterapia tiene como fin, utilizando diversas técnicas, obtener beneficios fisiológicos, sociales, psicológicos e intelectuales de los pacientes a los que se les aplica (Amorós, 2012), por lo tanto su utilización en demencias tipo Alzheimer, resulta ser una alternativa prometedora, aunque no se conocen todavía muchos datos de su eficacia.

En cualquier caso, por lo publicado hasta el momento, se sabe que sí genera una mejora en cualquier individuo, ya que disminuye la depresión (comentado anteriormente), la ansiedad que está asociada a un estado de estrés negativo, y aumenta el bienestar. En cuanto a la medida de esa mejora, existen alternativas biológicas como cuantificar el nivel de cortisol, alfa amilasa o inmunoglobulina A en saliva u orina pero, a pesar de que no son invasivas, estas técnicas si pueden aumentar el nivel de estrés de este tipo de paciente, con lo que se deberían de plantear otras alternativas que no generen esa situación que podría variar el estado emocional y por tanto el resultado de lo que se pretende medir. Una alternativa para valorar la mejora tras la intervención con algún tipo de terapia, podría ser la realización de una batería de preguntas que se encuadren en cuestionarios estandarizados. Estas preguntas deberían ser realizadas por los propios trabajadores del centro, de modo que los pacientes no se encontrasen desubicados. Para esto, previamente deberán ser aleccionados por los encargados de la investigación para conseguir que las preguntas no parezcan un interrogatorio, si no que se trate de una conversación coloquial, consiguiendo un ambiente de seguridad y confort para el enfermo.

\section{Objetivos}

En nuestro trabajo de investigación nos planteamos los siguientes objetivos:

Objetivo principal: Analizar la eficacia de la musicoterapia como una alternativa terapéutica no farmacológica en pacientes con demencia tipo Alzheimer.

Objetivo secundario: Valorar la utilización de cuestionarios estandarizados como un procedimiento eficaz para cuantificar el nivel de depresión, ansiedad, y bienestar de un paciente con demencia tipo Alzheimer.

\section{Hipótesis}

En relación a estos objetivos, nuestras hipótesis son:

Síntomas como la depresión, ansiedad y tristeza sufridos por los pacientes de Alzheimer disminuye tras la intervención mediante un protocolo de musicoterapia ralentizando el desarrollo de la enfermedad.

La aplicación de cuestionarios realizados en clave coloquial es una buena técnica para valorar esa mejora. 


\section{MATERIAL Y MÉTODO}

En este apartado describimos los diferentes aspectos relacionados con el material y la metodología empleada en el estudio.

\section{Diseño del estudio}

Se trata de un estudio prospectivo, mixto, analítico y experimental a través de un ensayo clínico por intención de tratar.

\section{Población}

Los candidatos a participar en el estudio serán adultos mayores de 65 años, institucionalizados en la Asociación Alzheimer Valencia (AFAV) afectos de DTA leve con GDS 4 y MMSE 18-23 inclusive, con trastornos de conducta y psicológicos.

La población final con la que queremos contar para realizar el estudio es de 25 pacientes que serán su propio control negativo.

\section{Procedimiento}

La propuesta del estudio a realizar sigue los siguientes pasos:

Se aplicarán los criterios de inclusión y de exclusión (detallados más adelante) obteniendo un listado definitivo de 25 pacientes.

Se recogerán los resultados de las escalas de valoración en esos pacientes, para lo cual se llevaran a cabo entrevistas diseñadas y estandarizadas, concretamente la de HADS (Hospital Anxiety and Depression Scale) (Carrobles, 1986) constituido por 14 ítems, 7 de depresión y 7 ansiedad (respuesta tipo Likert con 4 opciones posibles valoradas del 1 al 4) para valorar la depresión y ansiedad, y una versión del cuestionario MOOD (Rieffe, 2004) adaptada por Górriz, Prado-Gascó, Villanueva, Ordóñez y González, 2013, consistente en 4 estados de ánimo: Felicidad, Enfado, Tristeza y Miedo medido con 16 ítems en una escala de respuesta de tres puntos $(1=$ nunca, $2=a$ veces, $3=a$ menudo), de la que extrajimos las respuestas relacionadas con el estado de ánimo de la Felicidad (concretamente 4 ítems) para valorar el bienestar. En todas las dimensiones, puntuaciones mayores implican una mayor presencia de ese estado de ánimo.

A continuación, se aplicará el protocolo de Musicoterapia por grupos de 12-13 personas, a la misma hora (9:00 AM), detallado en el Anexo.

Tras la intervención con Musicoterapia, se realizarán las mismas entrevistas y por tanto se volverá a valorar a los pacientes participes del estudio con las mismas escalas y medidas que se utilizaron de forma previa a la intervención descritas anteriormente. 
El personal humano que realizará este estudio está formado por un equipo multidisciplinar en el que encontramos psicólogos, farmacéuticos, médicos, enfermeros y finalmente musicoterapeutas con una gran experiencia en ese campo.

\section{Criterios de selección}

En cuanto a los criterios de inclusión y exclusión, estos serán los siguientes:

Los criterios de inclusión:

Paciente con una edad mayor de 65 años, institucionalizado en una de la residencia de Valencia citada anteriormente.

Afectos de DTA con grado de severidad leve con GDS 4 o MMSE 18-23, presenten o no trastornos de conducta y/o psicológicos objetivados previamente.

Aceptación de los tutores en la participación del estudio.

Los criterios de exclusión:

Aquellos pacientes que muestren aversión a este tipo de terapias.

La no aceptación por parte de los tutores de su participación en el estudio.

\section{Análisis estadístico de los datos obtenidos}

El análisis de los datos del estudio consistirá en un contraste de hipótesis donde se valorará si las diferencias encontradas en las herramientas de medición antes-después de la intervención son estadísticamente significativas, con un margen de error de $0.05(p<0.05)$.

\section{Aspectos éticos}

Se contemplan los aspectos éticos expresados según la declaración de Helsinki y el informe Belmont, obedeciendo a los principios de justicia, no maleficencia y autonomía. Los autores garantizan la confidencialidad de los datos personales de las historias clínicas consultadas.

\section{RESULTADOS}

La muestra está conformada por 25 individuos cuya media de edad es de 77.52 años, con una desviación típica de \pm 7.37 años. De ellos 7 son hombres, que se corresponde con el $28 \%$ y 18 son mujeres que representa el $72 \%$.

Al evaluar la escala de HADS entorno a la ansiedad antes de la musicoterapia, obtuvimos que la puntuación media era de 19.83 con una desviación típica de \pm 2.54 . Mientras que tras la musicoterapia obtuvimos un 25.23 con una desviación típica de \pm 3.59 . Por otro lado, en la escala de depresión el valor medio previo a la musicoterapia fue de 20.50 con una desviación típica de \pm 4.77 , mientras que tras la musicoterapia 
aumento a 24.95 con una desviación típica de \pm 3.64 . Debemos recordar que a puntuación más elevada, menor es el grado de ansiedad y de depresión.

Tras ello, clasificamos tanto la ansiedad como la depresión en 4 niveles: normal, leve, moderado y grave. Hay que remarcar que las diferencias obtenidas en la depresión resultaron ser estadísticamente positivas $(p=0.007)$. A continuación, incluimos una síntesis de estos resultados (Tabla 1) para mejorar la compresión de los mismos.

Tabla1. Resumen de los resultados en \% obtenidos antes (PRE) y después (POST) del tratamiento, de los niveles de depresión y ansiedad

\begin{tabular}{ccccccc}
\hline & \multicolumn{2}{c}{ Normal } & \multicolumn{2}{c}{ Leve } & \multicolumn{2}{c}{ Moderada } \\
\cline { 2 - 6 } & PRE & POST & PRE & POST & PRE & POST \\
\hline Nivel de depresión & $40 \%$ & $72 \%$ & $28 \%$ & $8 \%$ & $12 \%$ & $0 \%$ \\
\hline Nivel de ansiedad & $28 \%$ & $80 \%$ & $64 \%$ & $0 \%$ & $4 \%$ & $4 \%$ \\
\hline
\end{tabular}

En cuanto al bienestar que generaba en esos pacientes la musicoterapia, esta lo extrapolamos a la felicidad sentida por los pacientes al recibir la terapia. Y al comparar los ítems del cuestionario MOOD relacionados con esta felicidad, antes y después de la musicoterapia, los resultados obtenidos fueron los siguientes (Tabla 2).

Tabla 2. Resumen de los resultados en \% obtenidos antes (PRE) y después (POST) del tratamiento, de los ítems relacionados con el estado de ánimo de la felicidad

\begin{tabular}{|c|c|c|c|c|c|c|c|}
\hline \multirow{2}{*}{ Ítems } & \multicolumn{2}{|c|}{ Nunca } & \multicolumn{2}{|c|}{ A veces } & \multicolumn{2}{|c|}{ A menudo } & \multirow[b]{2}{*}{$p$} \\
\hline & PRE & POST & PRE & POST & PRE & POST & \\
\hline Me he sentido feliz en las últimas 4 semanas. & $8 \%$ & $4 \%$ & $44 \%$ & $12 \%$ & $48 \%$ & $84 \%$ & 0.679 \\
\hline Me he sentido contento en las últimas 4 semanas. & $8 \%$ & $4 \%$ & $48 \%$ & $8 \%$ & $44 \%$ & $88 \%$ & 0.017 \\
\hline Me he sentido animado en las últimas 4 semanas. & $16 \%$ & $0 \%$ & $60 \%$ & $12 \%$ & $24 \%$ & $88 \%$ & 0.476 \\
\hline He sentido alegre en las últimas 4 semanas. & $8 \%$ & $4 \%$ & $36 \%$ & $8 \%$ & $56 \%$ & $88 \%$ & 0.147 \\
\hline
\end{tabular}

A pesar de que en todos los ítems hay una clara mejoría de la percepción de felicidad, sólo resulto ser estadísticamente positivo uno de ellos, representado por: "me he sentido contento en las últimas cuatro semanas", con $p=0.017$.

\section{CONCLUSIONES}

Las conclusiones que obtenemos tras nuestro estudio son que los beneficios de un protocolo corto de musicoterapia aplicado en pacientes con demencia tipo Alzheimer de grado leve son altamente satisfactorios, ya que se consigue disminuir en la mayoría de los pacientes, la depresión y la ansiedad relacionadas con el estrés, y se consigue aumentar su nivel de felicidad (estado de ánimo directamente relacionado con la sensación de bienestar). Este hecho se puede poner de manifiesto de un modo eficaz, y nada invasivo ni perjudicial para este tipo de pacientes, con cuestionarios estandarizados. En concreto nosotros hemos empleado el de HADS y el de MOOD, que se muestran como una buena herramienta para cuantificar estas mejorías, aunque pensamos que en un 
futuro se deben de replicar este tipo de estudios pero con una muestra poblacional mayor, profundizando de este modo en los beneficios de estas terapias.

\section{REFERENCIAS}

Amorós, B.Y. (2012). Musicoterapia en paciente oncológico. Cultura de los cuidados, 0, 57-73.

Andrés, V.P. (2000). El fenómeno de la música. En M.B. Toro, Fundamentos de musicoterapia (pág. 229). Morata.

Augé, P.M. (2000). Visión general y teoría de la musicoterapia. En M. B. Toro, Fundamentos de musicoterapia (pág. 289). Morata.

Carrobles, J.A., Costa, M., Del Ser, T. y Bartolomé, P. (1986). La práctica de la terapia de conducta. Valencia: Promolibro

Castro, M.R. (2005). El papel de la musicoterapia en los cuidados paliativos. Madrid: Medina.

Davis, W., Gfeller, K. y Thaut, M. (2000). Introducción a la musicoterapia. Teoría y práctica. Barcelona, Bolileau.

Díez, N. (1996). La terapia artística como una nueva enseñanza. Arte, individuo y sociedad (8), 2125.

Montés, J. (2013). Una reflexión sobre las terapias no farmacológicas y su aplicación desde la experiencia: 20 años del "Programa de atención a afectados de Alzheimer" de la asociación COTLAS. Información Psicológica (104), 84-99.

Rieffe, C., Meerum, M. y Bosch, J. (2004). Emotional awareness and somatic complaints in children. European Journal of Developmental Psychology, 1, 31-47.

Rodríguez, J.O., Ortiz, L.F., y Schwochert, R.M. (2012). Síntomas psicológicos y conductuales de la demencia: prevención, diagnóstico y tratamiento. Revista de Neurología (55), 598608.

Sagasti, T. y Bernaola, B. (1998). La Musicoterapia y la enfermedad de Alzheimer. Música, Arte y Proceso (6), 37-48.

Sánchez, C.T., Castro, F.V., Herrera, S.S. y Acuñas, M.G. (2013). Estado del arte sobre los efectos de la terapia asistida con perros en el tratamiento de enfermos de Alzheimer. International Journal of Developmental and Educational Psychology, 2(1), 271-283.

Sarasa, E. (2009). Terapia Ocupacional y Enfermedad de Alzheimer: Guía práctica para estimulación de los Servicios Sociales. Huesca: España.

Soria-Urios, G., Duque, P. y Moreno, J.M. (2011). Música y cerebro (II): evidencias cerebrales del entrenamiento musical. Revista de Neurología (53), 739-746.

Tárraga, L. (1998). Terapias blandas: programa de psicoestimulación integral, alternativa terapéutica para la persona con enfermedad de Alzheimer. Revista Neurológica (27), 5162. 


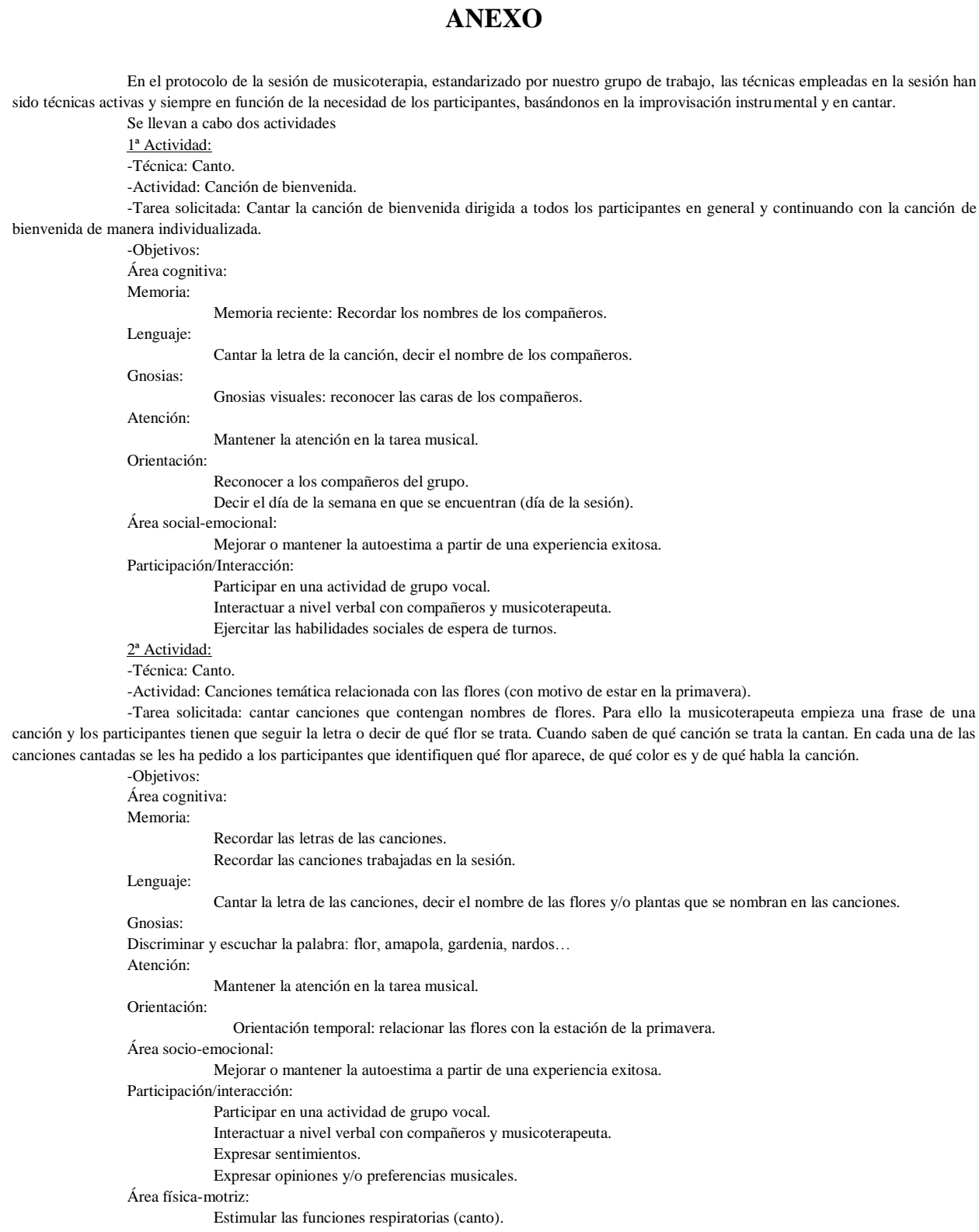
sido técnicas activas y siempre en función de la necesidad de los participantes, basándonos en la improvisación instrumental y en cantar.

Se llevan a cabo dos actividades

1 actividad:

-Técnica: Canto

-Actividad: Canción de bienvenida.

-Tarea solicitada: Cantar la canción de bienvenida dirigida a todos los participantes en general y continuando con la canción de bienvenida de manera individualizada.

-Objetivos:

Área cognitiva:

Memoria:

Memoria reciente: Recordar los nombres de los compañeros.

Lenguaje:

Gnosias:

Cantar la letra de la canción, decir el nombre de los compañeros.

Atención:

Gnosias visuales: reconocer las caras de los compañeros.

Orientación:

Mantener la atención en la tarea musical.

Área social-emocional:

Reconocer a los compañeros del grupo.

Decir el día de la semana en que se encuentran (día de la sesión).

Mejorar o mantener la autoestima a partir de una experiencia exitosa.

Participación/Interacción:

Participar en una actividad de grupo vocal.

Interactuar a nivel verbal con compañeros y musicoterapeuta.

2a Actividad:

Ejercitar las habilidades sociales de espera de turnos.

-Técnica: Canto.

-Actividad: Canciones temática relacionada con las flores (con motivo de estar en la primavera).

-Tarea solicitada: cantar canciones que contengan nombres de flores. Para ello la musicoterapeuta empieza una frase de una canción y los participantes tienen que seguir la letra o decir de qué flor se trata. Cuando saben de qué canción se trata la cantan. En cada una de las canciones cantadas se les ha pedido a los participantes que identifiquen qué flor aparece, de qué color es y de qué habla la canción.

-Objetivos:

Área cognitiva:

Memoria:

Recordar las letras de las canciones.

Recordar las canciones trabajadas en la sesión.

Lenguaje:

Cantar la letra de las canciones, decir el nombre de las flores y/o plantas que se nombran en las canciones.

Gnosias:

Discriminar y escuchar la palabra: flor, amapola, gardenia, nardos...

Atención:

Mantener la atención en la tarea musical.

Orientación:

Orientación temporal: relacionar las flores con la estación de la primavera.

Área socio-emocional:

Mejorar o mantener la autoestima a partir de una experiencia exitosa.

Participación/interacción:

Participar en una actividad de grupo vocal.

Interactuar a nivel verbal con compañeros y musicoterapeuta.

Expresar sentimientos.

Área física-motriz:

Expresar opiniones y/o preferencias musicales.

Estimular las funciones respiratorias (canto).

Recibido: 29 de abril de 2014

Recepción Modificaciones: 26 de mayo de 2014

Aceptado: 3 de junio de 2014 